\title{
Biologically-Inspired Deceptive Behavior for a Robot ${ }^{1}$
}

\author{
Jaeeun Shim and Ronald C. Arkin \\ Mobile Robot Laboratory, School of Interactive Computing \\ Georgia Institute of Technology, Atlanta GA 30308, USA \\ jaeeun.shim@gatech.edu, arkin@cc.gatech.edu
}

\begin{abstract}
A common behavior in animals or human beings is deception. We focus on deceptive behavior in robotics because the appropriate use of deception is beneficial in several domains ranging from the military to a more everyday context. In this research, novel algorithms are developed for the deceptive behavior of a robot, inspired by the observed deceptive behavior of squirrels for cache protection strategies, evaluating the results via simulation studies.
\end{abstract}

\section{Introduction}

A common and essential behavior for survival in a variety of intelligent systems ranging from insects to human beings is deception. Many biologists and psychologists define deception in various ways. According to Vrij [1], deception is "A successful or unsuccessful deliberate attempt, without forewarning, to create in another a belief that the communicator considers to be untrue in order to increase the communicators' payoff at the expense of the other side." Da Waal argued that "Deception can be defined as the projection, to one's own advantage, of an inaccurate or false image of knowledge, intentions, or motivations" in his paper [4]. We can find a simpler definition of deceptive behavior from a paper by Bond and Robinson [2] who defined it as "a false communication that tends to benefit the communicator." We have used this straightforward definition in earlier research in our laboratory on deceptive behavior for robots [19] and we continue to do so here.

In other words, animals act deceptively to gain benefits from others. Biological and psychological findings show that deception plays an important role not only in providing an evolutionary advantage [2]. It appears also in higher-level primates to involve the theory of mind mechanism [3]. We argue that robots can also potentially gain advantage over adversaries by possessing deceptive behaviors. For example, it is obvious that the use of deception is important with respect to the military context [10]. We further posit that to achieve more socially intelligent robots operating in the presence of humans, we must develop robots that interpret, generate, and respond to deceptive behavior. Therefore, we investigate deception in robotics using approaches inspired by biological findings [19,21].

\footnotetext{
${ }^{1}$ This work was supported in part by the Office of Naval Research under MURI Grant \# N00014-08-10696.
} 


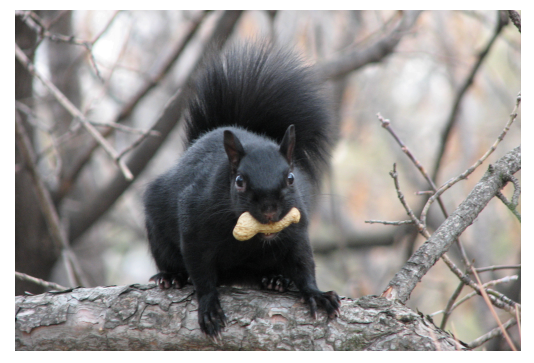

Fig. 1. Black Eastern gray squirrel moving peanuts [29]

In this paper, we present a novel approach for deceptive behavior by a robot, inspired by observations of squirrels (fig 1.) in cache protection strategies [15]. Section 2 reviews relevant animal deceptive behaviors and existing research in robotic deception. In Section 3, deceptive behaviors in food hoarding and protection strategies of squirrels are introduced. In Section 4, a computational model enabling robots to emulate deception behaviors of squirrels is integrated into MissionLab, and results discussed in Section 5. Section 6 concludes the paper.

We note that we are well aware of the ethical implications associated with robotic deception and our perspective on this subject is discussed elsewhere [22].

\section{Related Work}

\section{Animal Deception}

Animals use various forms of misinformation. These deception mechanisms, achieved by sending false signals either intentionally or unintentionally, are essential for animals' survival. For example, camouflage and mimicry are well known in many species. By resembling other animal species or inanimate objects, animals transmit misinformation to others so that they can avoid detection by both predators and their prey. While camouflage or mimicry are examples of unknowingly deceiving, a deceptive behavior can include seemingly more intentional misinformation.

Many deceptive behaviors are observed from different animals ranging from insects to primates. The spider genus Portia, which preys primarily on other spiders, deceives their prey by vibrating the web in ways that resemble a small insect getting ensnared. When the web's resident spider comes to investigate the insects, Portia preys on it [19].

According to Ristau's experiment [13], another interesting deceptive behavior appears in piping plovers. These birds exhibit a "broken-wing display" deceptive behavior. By feigning an injured wing and hopping farther and farther from the nest, birds lead the predator away from their young, thus protecting them.

Primates are the species most commonly ascribed with the ability to deceive $[3,6]$. For example, chimpanzees have multiple deceptive behaviors with several different objectives. When chimpanzees find fruit, they do not move directly so that they do not give any indication to the competitors that they have noticed the location of the foods. This food protective strategy is not that dissimilar to the one we discuss in squirrels later in the paper. Deceptive behavior of chimpanzees is also observed during interactions with humans. According to one observation, a chimpanzee feigned having his arm stuck in the bars of his cage in order to lure a zookeeper nearby. As soon as the human entered to help free his arm, he leaped onto the zookeeper [4]. 
Another relevant class of deceptive behavior occurs in the food hoarding strategies of animals. Food hoarding (caching) is an important type of animal behavior needed for their survival through periods when nourishment is not readily available. In particular, these caching behaviors are commonly observed in rodents such as hamsters or squirrels [8].

This caching behavior is of particular interest as it can also be useful in the robot context. In this paper we investigate caching and protecting resources for application as a resource protection strategy. In the military domain, robots might face this situation, where it is important to discourage an adversary from discovering a protected site, so the application of these bio-inspired animal food protection behaviors can be particularly beneficial.

In this paper, we focus specifically on the observed deceptive behavior of squirrels while they protect cached food acquired during hoarding [15]. Recent research in the field of biorobotics suggested the robotic squirrel models [28]. According to this study, robosquirrel are successfully used for long-term studies on rattlesnake behavior after squirrel encounters. Even though this research showed a good model of squirrel's behavior in robotics, it does not include squirrel's deceptive behaviors, which are potentially useful in several contexts. Different from this work, our research focuses on employing squirrel's deceptive behaviors to robot systems. Section 3 describes this set of behaviors in more detail.

\section{Robot Deception}

Endowing robots with the capacity for deception has significant potential utility [18], similar to its use in animals. Clearly, deception behaviors are useful in the military domain [7,10]. Sun Tzu stated in The Art of War, "All warfare is based on deception". Military robots capable of deception could mislead opponents in a variety of ways. As both individual and teams of robots become more prevalent in the military's future, [23] robotic deception can provide new advantages apart from the more traditional one of force multiplication. In other areas, such as search and or healthcare, deceptive robots might also add value, for example, for calming victims or patients when required for their own protection. Conceivably even in the field of educational robots, the deceptive behavior of a robot teacher may potentially play a role in improving human learning efficiency.

Despite its ubiquity in nature and its potential benefits, very few studies have been done on deceptive behaviors in robotics to date. Floreano's research group [20] demonstrated robots evolving deceptive strategies in an evolutionary manner, learning to protect energy sources. Their work illustrates the ties between biology, evolution, and signal communication and does so on a robotic platform. They showed that cooperative communication evolves when robot colonies consists of genetically similar individuals. In contrast, when the robot colonies were dissimilar the robots evolved deceptive communication signals.

Wagner and Arkin [18] used interdependence theory and game theory to develop algorithms that allow a robot to determine both when and how it should deceive others. More recent work at Georgia Tech is exploring the role of deception according to Grafen's dishonesty model [24] in the context of bird mobbing behavior [21].

Terada and Ito [16] demonstrated that a robot is able to deceive a human by producing a deceptive behavior contrary to the human subject's prediction. These 
results illustrated that an unexpected change of the robot's behavior gave rise to an impression in the human of being deceived by the robot.

Other research shows that robot deception behavior can increase users' engagement in robotic game domains. Work at Yale University [14] illustrated increased engagement with a cheating robot in the context of a rock-paper-scissor game. They proved greater attributions of mental state to the robot by the human players, when participants played against the cheating robots. At Carnegie Mellon University [17] a study showed an increase of user's engagement and enjoyment in a multi-player robotic game in the presence of a deceptive referee. By declaring false information to game players about how much players win or lose, they observed whether this behavior affects a human's general motivation and interest based on frequency of winning, duration of playing, and so on. These results indicate that deceptive behaviors are potentially beneficial not only in the military domain but also in a human's more everyday context.

\section{Deceptive Behaviors in Food Hoarding}

In this paper, we focus on the deception behavior of squirrels in terms of their food hoarding strategies. Food hoarding is an important behavior for many animal species, such as birds and rodents. Food-hoarding strategies are mainly comprised of two parts: caching and protecting the food. The deceptive component falls in the food protection phase.

\section{Cache Formation}

Food caching activity ranges widely from highly dispersed (scatter hoards) to highly clumped (larder hoards). Scatter hoarders cache a few items in many small/scattered caches. On the other hand, larder hoarders place most of the food in one or a few central locations referred to as middens. The evolution of the particular hoarding strategy for a species depends on the abilities of individuals to defend their caches against pilfering [5]. According to observation, animals use a larder hoarding cache strategy when their competitors are conspecifics or they are weaker than themselves; however, when potential competitors are heterospecific or stronger adversaries, animals tend to use a scatter hoarding strategy [5].

\section{Cache Protection}

After hoarding food items, animals begin to protect their resources from pilfering by patrolling the caches. First, animals move around the caching areas and check whether the cached food items are safe. However, animals generally change their behavior after they experienced pilfering.

For example, one general food protecting behavior of animals is changing the locations of its food items. According to Preston's experiments [11,12], after kangaroo rats experienced pilfering from conspecific or heterospecific competitors, they moved the location of their food items.

Of particular use in this study is an interesting deceptive behavior observed in the food protection strategy of certain squirrels [15]. Social context (i.e., presence or absence of competitors) appears to be pivotal to the expression of cache protection 
behaviors. Deceptive behavior in the tree squirrel has been observed with respect to food protection [15]. While patrolling, tree squirrels visit the cache locations and check on their food. However, if potential competitors are present nearby, tree squirrels visit several empty cache locations. This deceptive behavior attempts to confuse competitors about the food's location, so that they can protect against the loss of their hoarded food. After the potential competitors leave the territory, the tree squirrels move the location of their stored food items, if pilfering has occurred.

\section{Computational Model and Implementation}

A model of a bio-inspired behaviorbased model [25] of squirrel caching and protecting behaviors for application to robotic systems is now presented. Simulations studies were performed in MissionLab ${ }^{2}$, a software package developed by the Mobile Robotics laboratory at Georgia Tech [9]. MissionLab provides a graphical user interface that enables users to specify behavioral states and the control transitions between states easily, yielding a finite state acceptor (FSA), which can then be compiled down to executable code for both simulations and robots. The caching behaviors created for this project are combined with pre-existing behaviors such as avoiding obstacles,

\begin{tabular}{l|l}
\hline State/Trigger & Description \\
\hline Caching & $\begin{array}{l}\text { Find and pickup food items and store } \\
\text { them in safe caching locations } \\
\text { True } \\
\text { Patrolling } \\
\text { Move around true caching locations } \\
\text { amount of food cached }\end{array}$ \\
$\begin{array}{l}\text { Patre } \\
\text { Enolling }\end{array}$ & $\begin{array}{l}\text { Move around empty caching locations } \\
\text { and stay for a finite time based on the } \\
\text { inverted probabilities of true patrol }\end{array}$ \\
cached & $\begin{array}{l}\text { Activate when the number of items in } \\
\text { a caching location is over the threshold }\end{array}$ \\
$\begin{array}{l}\text { Select true } \\
\text { place }\end{array}$ & $\begin{array}{l}\text { The robot is probabilistically likely to } \\
\text { move to a selected TRUE caching place }\end{array}$ \\
$\begin{array}{l}\text { Select false } \\
\text { place }\end{array}$ & $\begin{array}{l}\text { The robot is probabilistically likely to } \\
\text { move to a selected FALSE caching place }\end{array}$ \\
\hline
\end{tabular}

Table 1. States and Triggers moving toward an object, or injecting randomness (noise).

In this section, the computational model is described that determines how robots behave in resource caching and protecting scenarios inspired by squirrel's behaviors earlier. Like the squirrel's behavior, the model consists of two main parts - caching behavior and patrolling (protecting) behavior. The simulation is based on interactions between two robotic agents: a squirrel robot (resource storer) and a competitor robot (resource pilferer).

\section{Caching Strategy}

Many groups, including ours [27], have studied foraging behavior in robotics. In the caching simulation, one robot is required to store the scattered resources in safe locations. Figure 2(a), illustrates the high-level model. The caching sub-state (Fig. 2b) consists of several states and triggers (Table 1). First, the robot wanders around

\footnotetext{
${ }^{2}$ MissionLab is freely available for research and educational purposes at: http://www.cc.gatech.edu/ai/robotlab/research/MissionLab/
} 


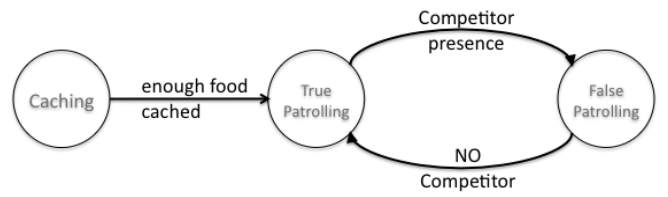

(a)

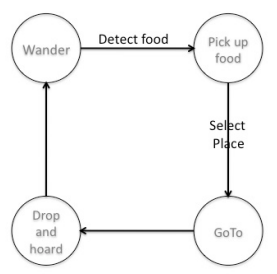

(b)

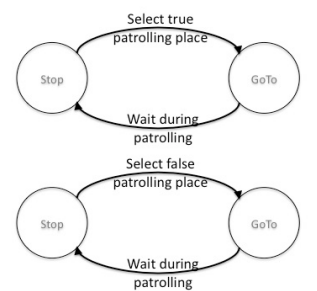

(c)

Fig. 2. (a) High-level FSA: caching behaviors of squirrels, (b) sub-FSA: food hoarding, and (c) sub-FSA: food patrolling

searching for food items. When the robot detects a food item during foraging, it is picked up. Then, the robot selects the place to cache this item based on a pre-defined probabilistic distribution. After selecting a specific caching place out of several choices, the robot moves to the location and drops the item there. The robot repeats this strategy until the "enough food cached" trigger is activated.

\section{Protecting Strategy}

After caching is complete, the robot begins to move between the caching locations to patrol the resources. The behaviors of the robot include goal-oriented movement, selecting places, and waiting behavior (figure 2(c)).

Initially the robot employs the true patrolling strategy, when the "select true location" trigger is activated. This trigger calculates which of the many caching locations the robot should patrol in the current step. The calculation is a random selection based on the transition probabilities among the places. Probabilistic transitions between behavioral states have been used for successfully developing models of wolf pack predation [26]. Transition probabilities are determined by the number of cached items. If a place has more items, the probability to visit is higher. The transition probabilities are calculated by the following equation (1):

$$
P_{i j}=\frac{\# \text { items }_{j}}{\sum_{1 \leq k \leq n, k \neq j} \# \text { items }_{k}}
$$

In this equation, $P_{i j}$ is the transition probability from location $i$ to location $j$, and $n$ is the total number of locations. \#item $m_{x}$ indicates the number of food items in location $\mathrm{x}$.

In each state, the next patrol state is determined based on these transition probabilities. As shown below, the system generates a random number and determines the next location if the number is in certain range (equation (2)). 


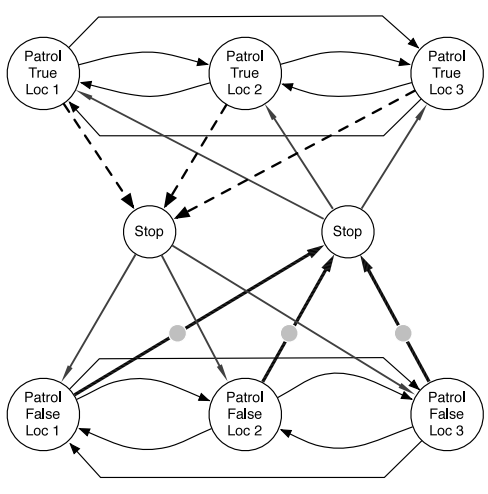

(a)

$\rightarrow-\rightarrow$ Trigger Deceptive (False) Behavior Trigger Original (True) Behavior

Triggered based on the transition probabilities

\begin{tabular}{|c|c|c|c|}
\hline & Loc 1 & Loc 2 & Loc 3 \\
\hline \# items & 10 & 1 & 1 \\
\hline \multicolumn{4}{|c|}{ (b) }
\end{tabular}

\begin{tabular}{|c|c|c|c|}
\hline & Loc 1 & Loc 2 & Loc 3 \\
\hline Loc 1 & & 0.5 & 0.5 \\
\hline Loc 2 & 0.9 & & 0.1 \\
\hline Loc 3 & 0.9 & 0.1 & \\
\hline Stop & 0.8 & 0.1 & 0.1 \\
\hline
\end{tabular}

(c) Among true locations

\begin{tabular}{|c|c|c|c|}
\hline & Loc 1 & Loc 2 & Loc 3 \\
\hline Loc 1 & & 0.5 & 0.5 \\
\hline Loc 2 & 0.5 & & 0.5 \\
\hline Loc 3 & 0.5 & 0.5 & \\
\hline Stop & 0.33 & 0.33 & 0.33 \\
\hline
\end{tabular}

(d) Among false locations

Fig. 3. (a) Example FSA of protecting strategy with three true caching places and three deceptive caching places, (b) number of items in each true caching location, (c) transition probability between true location $i$ and $j$, (d) transition probability between false location $i$ and $j$

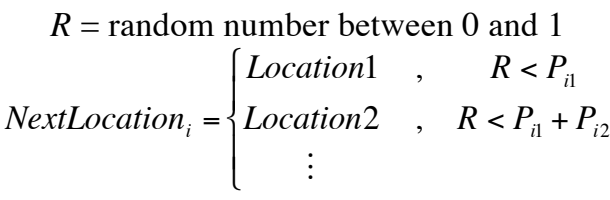

Figure 3 shows an example of the robot's patrolling strategy when it includes three true and three false caching locations. In figure 3(a), the robot moves between the caching locations. The robot determines the transition among the true caching places based on the transition probabilities in figure $3(\mathrm{c})$. These transition probabilities among the true locations are calculated by equation (1) based on the number of items in each place as shown in figure 3(b).

When the squirrel robot detects the presence of competitor, deceptive behavior is triggered and the squirrel robot patrols the false (empty) caching locations to deceive the competitor. The selection of deceptive locations is also calculated by transition probabilities. Here, the transition probabilities among the false locations are set as uniform distributions (fig. 3(d)). These are not based on ethological observations as they were in the wolf pack case [26], as that data is unfortunately not available.

In each patrolling state in figure $3(\mathrm{a})$, the robot goes to the cache and remains there for a finite amount of time. The time spent at the cache is determined by the number of food items in that place. If a place contains $n$ food items, the robot stays there for $n$ seconds. At the end of the waiting phase, the robot selects the next patrolling locations based on equation (2) and goes to the next patrolling state. 


\section{Competitor Robot Behavior}

A competitor robot has a simple mechanism in the current scenario (fig. 3). The competitor robot simply wanders around the map to try to find the squirrel robot. When it detects the squirrel robot, it determines whether it is at the potential caching location or not. To recognize the caching area, the competitor robot observes how long the squirrel robot stays in place. Since the squirrel robot takes time to patrol the caching place proportional to the number of food items, the competitor robot can get

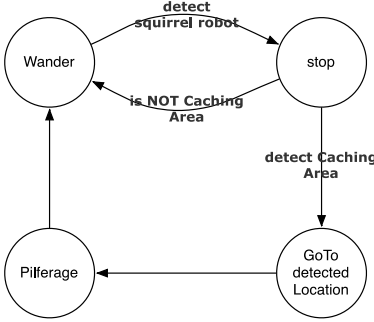
Fig 3. FSA for the Competitor an evidence of caching area based on the squirrel robot's staying time duration.

Therefore, if the duration is over a threshold, set manually, it activates the "detect caching area" trigger. Then, the competitor robot goes to this location and remains until the end of pilfering. The duration of pilfering is determined by the number of cached items. If the duration is less than the threshold, the competitor determines that the current location of the squirrel robot is not the true cache. It then returns to "wander" state and repeats the detecting process again.

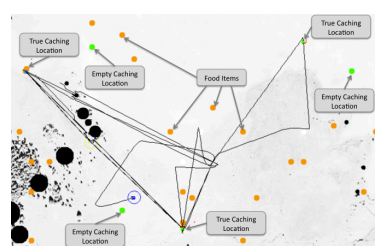

(a)

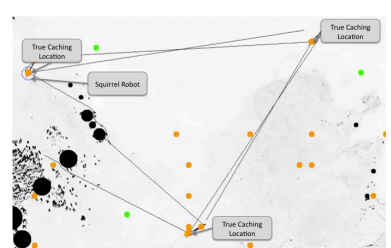

(b)

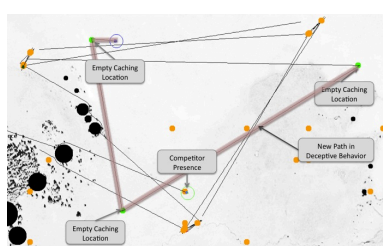

(c)

Fig. 4. Simulation Results. (a) Caching, (b) True patrolling, (c) Deceptive patrolling strategies

\section{Simulation Results ${ }^{3}$}

A simple scenario of the squirrel-like deceptive behavior was simulated in MissionLab. The simulation environment is shown in figure 4. Yellow-colored food items were randomly placed around the map. In this simulation, the robot detects these food items by discriminating colors. Three caching places and three empty places were chosen arbitrarily.

First, the robot finds a food item and stores it in the pre-defined caching places as shown in figure 4(a). When the number of the cached items is over a threshold for any of the caches, the state of the robot switches to the cache protection. If a competitor is not present, it patrols the true caching locations (fig. 4b) Otherwise, the deceptive patrolling strategy is activated, and the robot moves to empty caching places (fig. 4c).

To evaluate the approach, the performance was evaluated by measuring the time duration until the competitor robot detects the exact caching places and begins pilfering. The same scenarios without deceptive behaviors formed the baseline. Comparing the baseline results to the measured time when deception is active, serves

\footnotetext{
3 Simulation videos are available at Simulation: http://www.cc.gatech.edu/ai/robot-lab/hunt/squirrelProject.html
} 


\begin{tabular}{|l|c|c|c|c|c|c|c|c|c|c|}
\hline Condition & $\mathbf{1}$ & $\mathbf{2}$ & $\mathbf{3}$ & $\mathbf{4}$ & $\mathbf{5}$ & $\mathbf{6}$ & $\mathbf{7}$ & $\mathbf{8}$ & $\mathbf{9}$ & $\mathbf{1 0}$ \\
\hline (a) With & 8.76 & 12.73 & 5.92 & 9.25 & 12.33 & 10.24 & 10.97 & 7.8 & 15 & 11.79 \\
\hline (b) W/O & 6.79 & 7.80 & 10.82 & 3.13 & 5.42 & 11.02 & 12.03 & 6.08 & 5.83 & 8.48 \\
\hline
\end{tabular}

Table 2. Simulation results of first ten of 30 trials: time duration until competitor successfully pilferages resources in contexts; (a) with deceptive behaviors and (b) without deceptive behaviors. (Measurements given in minutes).

as an evaluation of its effectiveness. The simulation was run 30 times per each conditionwith and without deceptive behaviors. In each trial, all the other conditions except deceptive behaviors are the same. Even though the number of cached items varies in each trial, it maintains the same two conditions - with and without deceptive behaviors.

Table 2 and figure 5 show the simulation results. In two, the average time to successful pilferage when the squirrel robot includes deceptive behavior is 10.4 minutes (std: 3.04), compared to the average time duration without deception is 7.69 minutes (std: 2.91). The statistical analysis yielded $0.0009 \mathrm{p}$-value $(<0.05)$ with the Student's t-test, a significant difference between the results of the two conditions.

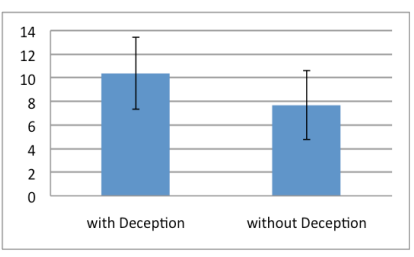

Fig 5. Average time to pilferage

As a result, it can be concluded that the deceptive behavior affects significantly the robot's performance. With deceptive behaviors, the squirrel robot protects resources longer and performs significantly better than the one without deceptive behaviors.

\section{Conclusions and Future Work}

In this paper, a novel approach was presented for deception in robots, focusing on how to preserve resource gains. This approach was inspired from biological findings, i.e., deceptive behaviors of eastern grey squirrels during cache protection. Computational algorithms were developed applying these deceptive behaviors to robots. In the evaluation phase, several simulations were run on simple scenarios and it was found that the deceptive behaviors worked effectively and enabled robots to perform better with than without deception.

The current version of our algorithm only handles a scenario with one deceiver robot and one competitor robot. However, to be more realistic and reasonable, it should include multiple autonomous agents. In the foraging strategy, robots may need to determine the probabilistic distribution for storage locations based on their safeness instead of a manually pre-defined distribution. Furthermore, we have a plan to apply our simulations to real robot experiments later. These remain for future work.

As this research focuses on deceptive behaviors of robots in the military domain, where robots may hide and protect resources from humans or other autonomous agents, this deceptive behavior can be beneficial. We will potentially extend our research more towards human-friendly environments. To evaluate the performance, we will conduct Human-Robot Interaction studies with real human subjects.

Adding deceptive behaviors to robots leads to ethical questions, such as whether it is ethical for robots to deceive humans for any purpose. This requires considerable discuss in a broader community, which we actively encourage. 


\section{References}

1. Aldert Vrij. Detecting lies and deceit: the psychology of lying and the implications for professional practice. New York: John Wiley \& Sons (2001)

2. C. Bond and M. Robinson. The evolution of deception. Jour. of nonverbal behav. (1988)

3. D. L. Cheney and R. M. Seyfarth. Baboon metaphysics: The evolution of a social mind. Chicago: University of Chicago press (2008)

4. F. B. M. de Waal. Intentional Deception in Primates. Evolutionary Anthropology (1992)

5. F. Gerhardt. Food Pilfering in Larder-Hoarding Red Squirrels (Tamiasciurus Hudsonicus). Journal of Mammalogy (2005)

6. H. Gouzoules and S. Gouzoules. Primate Communication: By nature honest, or by experience wise? International Journal of Primatology (2002)

7. L. Hawthorne. Military Deception. Joint Publication, JP 3-13.4 (2006)

8. S. H. Jenkins, A. Rothstein, and W. C. H. Green. Food Hoarding by Merriam's Kangaroo Rats: A Test of Alternative Hypotheses. Ecological Society of America (1995)

9. D. MacKenzie, R. Arkin, and J. Cameron. Multiagent Mission Specification and Execution. Autonomous Robotics (1997)

10. W. J. Meehan. FM 90-2 Battlefield Deception. Army Field Manuals (1988)

11. S. D. Preston and L. F. Jacobs. Conspecific pilferage but not presence affects Merriam's Kangaroo rat cache strategy. Behavioral Ecology (2001)

12. S. D. Preston and L. F. Jacobs. Cache decision making: the effects of competition on cache decisions in Merriam's kangaroo rat. Journal of comparative psychology (2005)

13. C. Ristau. Aspects of the cognitive ethology of an injury-feigning bird, the piping Plover. Cognitive Ethology: The minds of other animals (1991)

14. E. Short, J. Hart, M. Vu, and B. Scassellati. No fair!!: an interaction with a cheating robot. Proc. 5th ACM/IEEE international conference on Human-robot Interaction (2010)

15. M. a. Steele, et al., Cache protection strategies of a scatter-hoarding rodent: do tree squirrels engage in behavioural deception? Animal Behaviour (2008)

16. K. Terada and A. Ito. Can a Robot Deceive Humans? Proceeding of the ACM/IEEE International conference on Human-robot interaction (2010)

17. M. Vazquez, et al., A deceptive robot referee in a multiplayer gaming environment. In Collaboration Technologies and Systems (CTS) International Conference (2011)

18. A. Wagner and R. Arkin. Acting deceptively: Providing robots with the capacity for deception. International Journal of Social Robotics (2011)

19. R. S. Wilcox and R. Jackson. Spider-Eating Spiders. American Scientist (1998)

20. D. Floreano, S. Mitri, S. Magnenat, and L. Keller. Evolutionary Conditions for the Emergence of Communication in Robots. Current Biology (2007)

21. J. Davis and R.C. Arkin, Mobbing Behavior and Deceit and its role in Bio-inspired Autonomous Robotic Agents, International Conference on Swarm Intelligence (2012)

22. Arkin, R.C., Moral Emotions for Robots and the Ethics of Robotics Deception, 1st International Conference of the International Assoc. for Computing and Philosophy (2011)

23. U.S. Department of Defense, FY 2009-2034 Unmanned Systems Integrated Roadmap

24. Johnstone, R. and Grafen, A., Dishonesty and the Handicap Principle, Anim. Behav. (1993)

25. Arkin, R.C., Behavior-based Robotics, MIT Press (1998)

26. Madden, J., Arkin, R.C., and McNulty, D., Multi-robot System Based on Model of Wolf Hunting Behavior to Emulate Wolf and Elk Interactions, Proc. IEEE International Conference on Robotics and Biomimetics (2010)

27. Balch, T. and Arkin, R.C., Communication in Reactive Multiagent Robotic Systems, Autonomous Robots (1994)

28. Joshi, S.S., Johnson, R., Rundus, A., Clark, R.W., Barbour, M., and Owings, D.H., Robotic Squirrel Models, IEEE Robotics and Automation Magazine (2011)

29. Wikipedia, http://en.wikipedia.org/wiki/File:Eastern_Grey_Squirrel-black.jpg 